\title{
EARLY ENTERAL NUTRITION IS ASSOCIATED WITH BETTER OUTCOMES IN CHILDREN WITH SEVERE SEPSIS AND SEPTIC SHOCK
}

\author{
Sin Wee Loh¹, Judith Ju-Ming Wong², Wee Meng Han³, Chengsi Ong³, Yee Hui Mok², Jan Hau Lee² \\ 1 Department of Paediatric Medicine, KK Women's and Children's Hospital, Singapore \\ 2 Children's Intensive Care Unit, Department of Paediatric Subspecialties, KK Women's and Children's Hospital, Singapore \\ 3 Department of Nutrition \& Dietetics, KK Women's and Children's Hospital, Singapore
}

\begin{abstract}
INTRODUCTION
Positive clinical impact of adequate nutrition has been demonstrated in a heterogenous group of patients. Objectives of this study are to describe the nutritional practice in children with severe sepsis/septic shock admitted to our pediatric intensive care unit (PICU) and to determine the association between early nutrition (EN) and clinical outcomes.
\end{abstract}

\section{METHODS}

Retrospective study of patients admitted to PICU for sepsis/septic shock from January 2009 to April 2016. Demographics and daily nutritional data for the first 7 days of admission were collected. Outcomes of interest were PICU mortality, ventilation free days (VFDs) and PICU free days (IFDs).

\begin{tabular}{|l|l|}
\hline $\begin{array}{l}\text { Adequate } \\
\text { calories }\end{array}$ & $\begin{array}{l}\text { Receiving } \geq 80 \% \text { of resting energy } \\
\text { expenditure by third day of sepsis }\end{array}$ \\
\hline $\begin{array}{l}\text { Adequate } \\
\text { protein }\end{array}$ & $\begin{array}{l}\text { Receiving } \geq 1.5 \mathrm{~g} / \mathrm{kg} / \text { day of protein by third } \\
\text { day of sepsis }\end{array}$ \\
\hline Early EN & Initiation of EN within 48 hours of sepsis \\
\hline
\end{tabular}

\section{RESULTS}

There were 74 patients over the study period.

\begin{tabular}{|l|c|c|c|}
\hline Characteristics & Survivors (n=48) & $\begin{array}{c}\text { Non-survivors } \\
(\mathbf{n = 2 6})\end{array}$ & $\begin{array}{c}\text { p- } \\
\text { values }\end{array}$ \\
\hline $\begin{array}{l}\text { Age, median (IQR), } \\
\text { years }\end{array}$ & $11.1(4.9,13.5)$ & $8.2(3.6,12.5)$ & 0.16 \\
\hline Male gender & $23(47.9)$ & $12(46.2)$ & 0.89 \\
\hline $\begin{array}{l}\text { Weight, median } \\
\text { (IQR), kg }\end{array}$ & $30.7(16.5,51.0)$ & $25.3(13.5,34.3)$ & $\mathbf{0 . 0 3}$ \\
\hline $\begin{array}{l}\text { Types of infections } \\
\text { Bacterial }\end{array}$ & $9(18.8)$ & $10(38.5)$ & $\mathbf{0 . 0 2}$ \\
\hline $\begin{array}{l}\text { Viral } \\
\text { Fungi }\end{array}$ & $1(16.7)$ & $5(19.2)$ & \\
\hline $\begin{array}{l}\geq 2 \text { types } \\
\text { No identified } \\
\text { pathogens }\end{array}$ & $26(54.2)$ & $4(3.8)$ & \\
\hline Comorbidities & $21(43.8)$ & $21(80.8)$ & $<\mathbf{0 . 0 1}$ \\
\hline PIM2 score & $2.3(1.1,5.2)$ & $6.4(3.9,19.4)$ & $<\mathbf{0 . 0 1}$ \\
\hline ECMO & $0(0)$ & $3(11.5)$ & $\mathbf{0 . 0 4}$ \\
\hline $\begin{array}{l}\text { Inotropic support } \\
\text { Mechanical } \\
\text { ventilation }\end{array}$ & $39(81.3)$ & $26(100)$ & $\mathbf{0 . 0 2}$ \\
\hline 18 & $25(96.5)$ & $\mathbf{0 . 0 1}$ \\
\hline
\end{tabular}

IQR: Interquartile range, ECMO: Extracorporeal membrane oxygenation, PIM2: Pediatric index of mortality 2 score

Categorical variables were described in absolute numbers (percentages)

Continuous variables were described in medians (IQR)

\begin{tabular}{|c|}
\hline Nutrition was suboptimal \\
- $\quad$ Protein adequacy was $4 / 74(5.4 \%)$ and caloric \\
adequacy was $8 / 74(10.8 \%)$ \\
$26 / 74(35.1 \%)$ had early EN \\
- $\quad 26 / 45(53 \%)$ of patients started on EN had feeds \\
interruption of $\geq 6$ hours
\end{tabular}

Early EN was associated with better clinical outcomes

Multivariate analysis

\section{Early EN was associated with}

1. Decreased mortality: Adjusted OR $0.16(95 \% \mathrm{Cl}$ $0.04-0.67), p=0.01$

2. Increased VFDs: $\beta 9.01(95 \% \mathrm{Cl} 3.43-14.74)$, $p<0.01$

3. Increased IFDs: $\beta 9.80(95 \% \mathrm{Cl} 4.89-14.72)$, $p<0.01$

\section{DISCUSSION}

- Our study showed that suboptimal nutrition was prevalent in children with severe sepsis or septic shock.

- We identified an association between early EN and improved mortality rate, which was similar to a cohort study done on children with ARDS.

- Limitations:

(i) Might not have adjusted for unknown confounders

(ii) Small sample size

(iii) Retrospective study

- These results support the importance of aggressive nutrient delivery in the care of critically ill children.

\section{- Suboptimal nutrition was common in our} cohort.

- Early EN was associated with improved clinical outcomes.

- It is important to prioritise nutrition in critically ill children.

References

Mehta NM et al. Guidelines for the Provision and Assessment of Nutrition Support Therapy in the Pediatric Critically III Patient: Society of Critical Care Medicine and American Society for Parenteral and Enteral Nutrition. Pediatr Crit Care Med. 2017;18(7):675-715

2. Wong JJ et al. Nutrition Delivery Affects Outcomes in Pediatric Acute Respiratory Distress Syndrome. JPEN J Parenter Enteral Nutr. 2017;41(6):1007-13.

Mehta NM et al. Nutritional practices and their relationship to clinical outcomes in critically ill children--an international multicenter cohort study*. Crit Care Med. 2012;40(7):2204-11. 